\title{
Überseeische Verfassungsvergleichung nach 30 Jahren
}

\author{
Von Brun-Otto Bryde
}

\section{1. Überseeische Verfassungsvergleichung 1968}

Die Wahl eines Zeitrahmens von 30 Jahren im Titel meines Vortrages ist durch den Anlaß unserer Zusammenkunft, die Gründung der Zeitschrift "Verfassung und Recht in Übersee" 1968, bestimmt. Natürlich ist die Geschichte "überseeischer Verfassungsvergleichung" älter. Auch wenn man den Begriff nicht rein geographisch versteht und die USA aus der Betrachtung ausschließt, beginnt die Beschäftigung mit überseeischen Verfassungen entsprechend den jeweiligen Entkolonisierungsschüben für Lateinamerika vor über 100, für Asien vor 50 und für Afrika vor fast 40 Jahren. Aber die Gründung der Zeitschrift vor 30 Jahren war kein Zufall. Auch wenn sie für Deutschland damals bahnbrechend war, (und ziemlich lange allein auf breiter Flur blieb, bis das Jahrbuch für Öffentliches Recht sich unter der Herausgeberschaft Peter Häberles ${ }^{1}$ verstärkt unseres Themas annahm), in internationaler Perspektive liegt das Gründungsjahr der Zeitschrift, 1968, mitten in der ersten Entwicklungsdekade, als auch in der internationalen Rechtswissenschaft eine Aufbruchszeit einsetzte. Diese wird vielleicht am besten mit dem Begriff der "Law and Development"Bewegung gekennzeichnet. ${ }^{2}$ Zwar gab es insbesondere in den alten Kolonialländern Vorläufer (Kolonialrecht; droit d'outre mer; auch am Beginn der Hamburger juristischen Fakultät steht ja das Kolonialrecht). Aber solche traditionelle Überseerechtsforschung wurde in dieser Zeit umgestaltet, in theoretische Beziehung zu Modernisierung und Entwicklung gebracht, und vor allem in den USA wurde mit Nachdruck daran gearbeitet, auch den Juristen ihren Platz in der damals anhebenden Konjunktur der Entwicklungswissenschaften zu verschaffen. ${ }^{3}$ Herbert Krüger versuchte mit der Gründung eines Referats für "Verfas-

Die Verbundenheit der beiden deutschen Initiativen auf diesem Gebiet wird durch die erste Herbert-Krüger-Gedächtnisvorlesung dokumentiert: $P$. Häberle, Die Entwicklungsländer im Proze $B$ der Textstufendifferenzierung des Verfassungsstaates, VRÜ 1990, S. $225 \mathrm{ff}$.

B.-O. Bry'de, Die Rolle des Rechts im EntwicklungsprozeB, in: ders./F. Kübler (Hrsg.), Die Rolle des Rechts im EntwicklungsprozeB, 1986, S. 9, 11 ff. m.w.N.; Trubek/Galanter, Scholars in SelfEstrangement, Wisconsin Law Rev. 1974, S. 1062 ff.; Merryman, Comparative Law and Social Change: On the Origins, Style, Decline and Revival of the Law and Development Movement, Am. J. Comp. L 25 (1977), S. 457 ff.; Burg, Law and Development: A Review of the Literature and a Critique of "Scholars in Self-Estrangement", Am J.Comp.L 25 (1977), S. 492 ff.

3 Vgl. z. B. Wilken, A Glorious Opportunity for American Lawyers, A.B.A.J. 47 (1961), S. 142 ff.; weitere Nachweise bei Bryde (Fn. 2), S. 12 und Gardner, Legal Imperialism - American Lawyers and Foreign Aid in Latin America 1980, S. 35 ff. 
sungsentwicklung und internationale Beziehungen der neuen Staaten in Übersee" an der damaligen Forschungsstelle für Völkerrecht und ausländisches öffentliches Recht (dem in damals noch möglichen optimistischen Planungen zwei weitere folgen sollten, so daß alle drei Entwicklungskontinente angemessen hätten bearbeitet werden können), der Gründung der Zeitschrift und der Vergabe von Dissertationen einen vergleichbaren Aufbruch anzustoßen: vergeblich, wie wir heute wissen. Das Fach ist in der deutschen Rechtswissenschaft marginal geblieben.

Für die Verfassungsvergleichung schuf die letzte und umfassendste Entkolonisierungsbewegung dieser Jahre vor allem umfangreiches neues Material. Dutzende neue Verfassungen boten sich als Buch- oder Aufsatzthema an: Exemplarisch etwa aus dem ersten Heft der Auf satz des ersten Chef redakteurs Dieter Schröder über die Bundesstaatlichkeit in Nigeria ${ }^{4}$, oder, bis heute verfassungshistorisch interessant, das erste Beiheft der Zeitschrift von Enno Kliesch über den Einfluß des französischen Verfassungsdenkens auf afrikanische Verfassungen. 5

\section{Modernisierung durch Verfassungsrezeption}

Als Herbert Krüger in der ersten Nummer das Programm umriß, ${ }^{6}$ war eine erste sehr naive Stufe deutscher Beschäftigung mit den neuen Staaten schon überwunden: Bloßer Deskription oder allzu ethnozentrischen Konzepten "unvollendeter Demokratien" ${ }^{7}$ setzte Krüger den noch immer lesenswerten Versuch entgegen, das von ihm selbst repräsentierte Erbe klassischer deutscher Staatslehre mit angelsächsischer Politikwissenschaft zu verbinden. Ein Programm, das zwar an das damals herrschende Modernisierungsparadigma gebunden war, aber - was sich als fruchtbar erweisen sollte - sehr offen für eigenständige, unvorhersehbare Entwicklungen im Beobachtungsraum blieb. Man sollte nicht in falscher Pietät dem Gründungsvater gegenüber behaupten, er sei in allem ein besonders guter Prophet gewesen. Die Leistungsfähigkeit des neuzeitlichen Staatsmodells wird ebenso überschätzt wie der Säkularismus der Entwicklungsgesellschaften. Aber damit befand er sich damals in bester Gesellschaft: Die Vorstellung, unterentwickelte Gesellschaften ließen sich durch die Rezeption von Recht und Verfassung aus dem Norden modernisieren, war Allgemeingut gerade in der Law-and-Development-Diskussion. ${ }^{8}$ Dabei wäre es allerdings falsch zu glau-

4

5

E. Kliesch, Der Einfluß des französischen Verfassungsdenkens auf afrikanische Verfassungen, 1976.

6 H. Krüger, Verfassung und Recht in Übersee, VRÜ 1968, S. 3 ff.

7

8

Bryde (Fn. 2), S. $14 \mathrm{ff}$. 
ben, daß der Einführung westlicher Verfassungsmodelle im Zuge der Entkolonisierung auf Seiten der Akteure ein entwickeltes theoretisches Modell vom Zusammenhang wirtschaftlicher Entwicklung und westlichem Verfassungsrecht zugrunde lag. Das galt vielleicht am ehesten für die wenigen Fälle freiwilliger Rezeption (Japan, kemalistische Türkei ). Wo neue Verfassungen im Zuge der Dekolonisierung entstanden, übertrugen die Kolonialmächte ihr eigenes Modell, sicher auch weil sie es ehrlich für das beste hielten, vor allem aber, weil es national geprägten Juristen, und das gilt bis heute gerade auch in Deutschland, ohnehin schwer fällt, sich ein anderes System als das eigene auch nur als denkmöglich vorzustellen.

Aber in der Wissenschaft war die Annahme, daß das Modell westlicher Verfassungsstaatlichkeit in seinen jeweiligen Ausgestaltungen nicht nur notwendige, sondern auch hinreichende Bedingung für die Modernisierung der Gesellschaft sein würde, durchaus verbreitet. Dabei wurde durchaus gesehen, daß das tatsächliche Funktionieren eines solchen Verfassungstransfers zu wünschen übrig ließ, aber man ging noch von der Entschlossenheit "modernisierender Eliten"9 aus, dem Verfassungsstaat auf dem Papier eine verfassungsstaatliche Demokratie nachwachsen zu lassen. Karl Loewenstein hat in seiner Trias von Einteilungen von Verfassungen nach ihrem Realitätsbezug ("normative, nominalistische und semantische Verfassungen"10) eine ganze Kategorie, die der "nominalistischen" Verfassung, auf diese (Fehl-)Annahme gestützt: Als nominalistisch bezeichnet er nämlich Verfassungen, die zwar (noch) ineffektiv sind, aber vom guten Willen der Machthaber getragen werden, sie effektiv zu machen. ${ }^{11}$

\section{Vom Realismus zum Defätismus: Bankrott überseeischer Verfassungsvergleichung?}

Diese Hoffnungen erfüllten sich nicht. Schneller als auf anderen Rechtsgebieten erwiesen sich die rezipierten Verfassungen in allzu vielen Staaten als lebensunfähig.

Als ich Mitte der 70er Jahre im Rahmen meines Buches über "The Politics and Sociology of African Legal Development" eine Bestandsaufnahme über 43 afrikanische Regierungssysteme vornahm, waren 19 Militärregime, nur zwei (Botswana und Gambia) hatten am Westminstermodell festgehalten, der Rest waren überwiegend Einparteiensysteme, von

Apter, Politics of Modernization, 1969; dazu H. Krüger (Fn. 6), S. 26 ff.

$K$. Loewenstein, Reflexions sur la valeur des constitutions dans une époque revolutionnaire, Rev. francaise de science politique 1952, S. 5 ff., 312 ff.; ders., Verfassungslehre, S. $151 \mathrm{ff}$.

11

Zur Kritik Bryde, Verfassungsentwicklung, 1982, S. 28 Fn. 4. 
denen nur wenige (Tanzania und Kenya) als relativ partizipatorisch ein gewisses Maß an freier politischer Betätigung erlaubten. ${ }^{12}$

In anderen Kontinenten war es nicht anders. In Lateinamerika übernahmen die Militärs mit dem dramatischen Höhepunkt im Sturz Allendes auch bis dahin als relativ stabil angesehene Demokratien, in Asien blieben nur Indien und Japan den Unabhängigkeits- bzw. Nachkriegsverfassungen treu, auch hier breitete sich das Militärregime aus (Korea, Pakistan, Indonesien, Burma).

$\mathrm{Da}$ die Entkolonisierungsverfassungen wenig Bestand hatten, ist nicht erstaunlich, man mag sich allenfalls fragen, warum der Verfassungstransfer so sehr viel weniger erfolgreich war, als der Rechtstransfer auf anderen Rechtsgebieten. Denn in den meisten Staaten blieb das kolonial rezipierte Recht ja nicht nur erhalten, sondern die Verwestlichung des Rechtssystems beschleunigte sich in der Regel sogar mit der Unabhängigkeit, weil die neuen Eliten eher weniger Sympathie für "traditionelle" Rechte hatten, als die kolonialen Administratoren, deren Ziel es häufig war, "to keep the natives native". ${ }^{13}$ Aber auf anderen Rechtsgebieten erfolgte nicht nur auf dem Papier, sondern auch soziologisch ein wirklicher, wenn auch unvollkommener Rezeptionsprozess. Die Widerstandskraft des importierten Rechts nach der Unabhängigkeit lag gerade darin, daß es nicht einfach nur von der Kolonialmacht oktroyiert, sondern von einem einheimischen Juristenstand zur eigenen Rechtstradition gemacht worden war. ${ }^{14}$ Die am Unabhängigkeitstag in Kraft tretende Verfassung baute nicht auf einem vergleichbaren Rezeptionsprozeß auf: Ein koloniales System ist seiner Natur nach autoritär, auch wenn es dort, wo der Prozeß nicht allzu überstürzt verlief, eine Übergangsphase "kontrollierter" Demokratie mit lokaler Beteiligung in Legislative Councils und ähnlichen Einrichtungen gab. Die Kolonialmächte hatten die neu entstehenden Staaten also nicht auf Demokratie und Verfassungsstaatlichkeit vorbereitet und die durchaus verbreitete Vorstellung "Wir haben eine so schöne Demokratie hinterlassen, aber sie konnten nicht damit umgehen" ist falsch. Ganz im Gegenteil konnten die bald entstehenden diktatorischen Systeme nahtlos an das Kolonialrecht anknüpfen, z.B. ihre Macht mit Hilfe von "Preventive Detention Acts" sichern, die aus dem kolonialen Recht in das der neuen Staaten übernommen worden waren. ${ }^{15}$ Aber auch die übernommenen Verfassungsmodelle ließen sich verhältnismäßig leicht autoritär instrumentalisieren. Für das frankophone Afrika z.B. ist es nicht ohne Folgen geblieben, daß das auf das persönliche Regiment

12

13

14

15

Bryde, The Politics and Sociology of African Legal Development, 1976, S. $23 \mathrm{ff}$.

Bryde (Fn. 12), S. 9 ff. m.w.N.

Bryde, Rezeption europäischen Rechts und autozentrierte Rechtsentwicklung in Afrika, Afrika

Spectrum 1977, S. 117 ff.; ders. (Fn. 2), S. 16 ff.

Aktuelles Beispiel ist Kenia, vgl. auch Bryde (Fn. 12), S. 14. 
de Gaulles zugeschnittene Verfassungssystem der V. Republik das Vorbild war: ${ }^{16}$ Es bedurfte nicht sehr viel Anpassung um aus einem exekutiven Präsidialsystem ein autoritäres zu machen. ${ }^{17}$ Und in ehemals britischen Gebieten mußte man nur die Königin durch einen Präsidenten ersetzen und die ungeschriebenen Konventionen, die das Staatsoberhaupt im britischen System bei der Ausübung seiner Befugnisse binden, weglassen, um eine Präsidialdiktaur zu errichten. ${ }^{18}$

Jedenfalls entwickelten sich die "unvollendeten Demokratien" entgegen den Hoffnungen der Modernisierungstheoretiker nicht in vollendete, und zunehmend autoritäre Systeme erwiesen sich nicht als "Erziehungsdiktaturen", sondern viel häufiger als "Kleptokratien", ${ }^{19}$ das heißt, Systeme zur Bereicherung des Staatschefs und seiner Entourage. Der klassische Beispielsfall, der diesem System in der Politikwissenschaft den Namen gegeben hat, Mobutus Herrschaft in Zaire, ist gerade dieses Jahr zu Ende gegangen. Die Verbreitung solcher Systeme, insbesondere des Militärregimes, in historisch und kulturell ganz unterschiedlich geprägten Teilen der Dritten Welt spricht dafür, daß ein Zusammenhang mit "Unterentwicklung" besteht. Allerdings nicht mit mangelnder demokratischer Reife einer rückständigen Bevölkerung, wie im Norden vorschnell und mit bemerkenswert wenig Reflexion der eigenen Geschichte angenommen, aber auch von den Regierenden im Süden gerne behauptet wird. Dagegen sprechen schon die wenigen Ausnahmen und Zwischenspiele, in denen die analphabetische Masse durchaus sinnvoll mit dem Stimmzettel umzugehen wußte. Sowohl der Sturz Indira Ghandis nach ihrer autoritären Herrschaft in der emergency wie ihre Rückkehr, als sich ihre Nachfolger als regierungsunfähig erwiesen, sind Beispiele. Der Zusammenhang besteht vielmehr zwischen wirtschaftlicher Krise und politischem System: Die überragende Bedeutung der wirtschaftlichen Lage für die Wahlentscheidung ist in der Politikwissenschaft gut dokumentiert. Überträgt man das auf Entwicklungsgesellschaften, muß man einen Regierungswechsel bei jeder Wahl erwarten, was angesichts der Ressourcen, über die die Regierenden verfügen können, für diese schwer akzeptabel ist und die Versuchung, die Machtmittel des Amtes zur Verlängerung des Amtes zu nutzen, sehr groß macht. ${ }^{20}$ Trotz Lippenbekenntnissen zu Demokratie und Menschenrechten ziehen aber auch ausländische Investoren und internationale Fininzagenturen autoritäre Stabilität demokratischer Unruhe vor. ${ }^{21}$

16

17

18

19

20

21

Kliesch (Fn. 5).

Bryde (Fn. 12), S. 20.

Bryde, ebendort.

Körner/Maaß/Siebold/Tetzlaff, Im Teufelskreis der Verschuldung, 1978, S. 55.

Zum Zusammenhang von Diktatur und Unterentwicklung vgl. Bryde (Fn. 2), S. 28 ff.; Leftwich, On the Primacy of Politics in Development, in: ders., Democracy and Development, 1996, S. 17 ff.

Vgl. auch Leftwich (Fn. 21), mit dem Nachweis, daß die demokratischen Ausnahmen sich durch große faktische Stabilität auszeichneten: S. 19. 
Jedenfalls war, von wenigen Ausnahmefällen abgesehen, in dieser Zeit die Verfassungswirklichkeit so wenig durch normative Verfassungen geprägt, daß die überseeische Verfassungsvergleichung Schwierigkeiten mit ihrem Gegenstand bekam. Das bedeutet nicht, daß nicht auch die Beschäftigung mit den in dieser Zeit entstehenden Verfassungssystemen von Interesse gewesen wäre. Während die Exegese bloß auf dem Papier stehender "symbolischer" bzw. "semantischer" Verfassungen, ${ }^{22}$ wie es sie insbesondere in Lateinamerika gab, in der Tat ziemlich unf ruchtbar ist, entstanden in dieser Zeit in Afrika neuartige Verfassungssysteme, denen jedenfalls Originalität und Ehrlichkeit nicht abzusprechen war: Vor allem das des nicht einfach nur durch politischen Druck und Manipulation durchgesetzten, sondern verfassungsrechtlich institutionalisierten Einparteienstaates. Daß dieser Verfassungstyp des "demokratischen Einparteienstaates", der eine Zeitlang geradezu als afrikanischer Beitrag zur Verfassungsvergleichung erschien, gleichzeitig mit dem Zusammenbruch der realsozialistischen Systeme in Osteuropa verschwand und häufig mit einem Modell "afrikanischer Sozialismus" verbunden war, läßt möglicherweise übersehen, daß solchen Systemen, denen Rogge eine schöne Dissertation gewidmet hat, ${ }^{23}$ im Ansatz jedenfalls in einigen Fällen ein innovatives Konzept zugrunde lag. Für Julius Nyerere etwa war die Rede vom "demokratischen" Einparteiensystem keine Heuchelei: Er sah in einer Situation, in der bei Wahlen nach dem von Großbritannien ererbten Mehrheitswahlrecht die Unabhängigkeitspartei alle Sitze gewann, ein System, in dem mehrere Kandidaten der Einheitspartei gegeneinander antraten, als demokratischere Alternative an. ${ }^{24}$ Die Möglichkeiten, und später dann natürlich auch Probleme der Nutzung einer solchen Partei als Mobilisierungsund Erziehungsinstrument kamen hinzu. Schließlich ist gerade in Afrika - aber mit dem Zusammenbruch des Kongresses und Aufkommen religiöser und ethnischer Parteien in Indien auch anderswo (und Europa kennt diese Phänomen natürlich auch: Nordirland) das Problem der Verstärkung ethnischer und religiöser Gegensätze in einem Mehrparteiensystem bis heute ungelöst. ${ }^{25}$ Demokratisierungsversuche nach dem Ende von Diktaturen (in Uganda, im Kongo) experimentieren daher eher mit "Kein-Parteien-Systemen" als mit Mehrparteiensystemen.

Auch das Militärregime in der Dritten Welt, dem Herbert Krüger seine letzte, in meiner Sicht viel zu positive Monographie gewidmet hat, ${ }^{26}$ verdiente die Beachtung der Verfas-

22

23

24

25

26

Zur Begrifflichkeit: Bryde, Verfassungsentwicklung, 1982, S. 27 ff.; Loewenstein (Fn. 10).

Rogge, Die Verfassung des afrikanischen Einparteienstaates, 1974.

Nyerere, Demokratie und Parteiensystem, in: Afrika heute, 1963, Dok. 1963.

Nach der gerade in Deutschland verbreiteten Auffassung, daß es Demokratie nur in homogenen Staatsvölkern geben kann (exemplarisch: E.W. Böckenförde, Demokratie als Verfassungsprinzip, HdbStR I, 1987, S. 906, 918), dürfte es fast nirgendwo in der Dritten Welt Demokratien geben; zur Kritik: Bryde, Die bundesrepublikanische Volksdemokratie als Irrweg der Demokratietheorie, Staatswissenschaft und Staatspraxis, 1994, S. 305, 310 ff. m.w.N.

H. Krüger, Militärregime in Übersee, 1976. 
sungsvergleichung. Wiederum gibt es auch juristisch interessante Besonderheiten, wie z.B. das bundesstaatliche Militärregime in Nigeria, die mit der bloßen Konstatierung einer Herrschaft einer Offiziersclique nicht erledigt sind. Evers' Dissertation über die Militärregierung in Argentinien ist ein gelungenes Beispiel für eine politisch sensible juristische Bearbeitung dieses Themas. ${ }^{27}$

Trotzdem ist die Frustration des Verfassungsvergleichers angesichts der Konfrontation mit einer nicht verfassungsstaatlichen Wirklichkeit mit Händen zu greifen. Es erwies sich daher als Glücksfall für die Zeitschrift, daß Herbert Krüger, seinem eigenen "katholischen" Verfassungsverständnis verpflichtet, das weder eine Beschränkung der Rechtswissenschaft auf das positive Recht akzeptierte noch ein solches der Verfassung auf Verfassungsrecht, ${ }^{28}$ von vorneherein auf die Einbeziehung sowohl der Sozialwissenschaften wie anderer Rechtsgebiete, vor allem auch des internationalen Rechts angelegt war. Die Zeitschrift konnte daher in dieser Zeit, als eine Beschäftigung nur mit dem positiven Verfassungsrecht der überseeischen Kontinente ziemlich frustrierend gewesen wäre, auf andere Themen und Sozialwissenschaften ausweichen, was fruchtbar war und nicht rückgängig gemacht zu werden braucht.

\section{Demokratisierung: neuer Start oder Wiederholung der alten Fehler ?}

Seit einigen Jahren erleben wir nun eine neue Welle demokratischer und rechtsstaatlicher Verfassungsgebung, vor allem natürlich in Osteuropa nach dem Zusammenbruch des kommunistischen Systems, aber zeitgleich und in Verbindung damit auch in allen Entwicklungskontinenten. Huntington hat in diesem Zusammenhang von der dritten Demokratisierungswelle gesprochen (wobei er die erste mit französischer und amerikanischer Revolution, die zweite nach dem zweiten Weltkrieg, und die noch immer andauernde dritte mit der Nelkenrevolution in Portugal beginnen läßt). ${ }^{29} 1973$ waren nur $25 \%$ der Staaten jedenfalls formal demokratisch, inzwischen liegt die Zahl bei ca. $70 \%{ }^{30}$ Vor allem in Lateinamerika wurden Militärregime und autoritäre Diktaturen in einem umfassenden Prozeß abgelöst, aber auch Afrika sah das Ende des Apartheidsystems und vielerorts die Abkehr vom Einparteienstaat und Präsidentialismus. In Asien wurden in wichtigen Staaten (Süd-Korea, Thailand) Militärregime abgelöst, der Prozeß ist noch im Gange (Indonesien, Burma). Das

27

T. Evers, Militärregierung in Argentinien - Das politische System der "Argentinischen Revolution", 1972; vgl. auch ders., Die "Gesetzesdekrete" argentinischer Revolutionsregierungen, VRÜ 1968, S. $333 \mathrm{ff}$.

28

29

30

Krüger (Fn. 6), S. 6 f.

S.P. Huntingdon, The Third Wave: Democratization in the Late Twentieth Century, 1991.

A. Leftwich, On the Primacy of Politics in Development, in: ders., Democracy and Development, 1996, S. 3. 
Bild sollte dennoch nicht übertrieben optimistisch gezeichnet werden. Rückschläge sind an der Tagesordnung (in jüngster Zeit in Sierra Leone und in Congo-Brazzaville). ${ }^{31}$ Trotzdem ist die Gesamttendenz bemerkenswert und bedeutet für die überseeische Verfassungsvergleichung zunächst einmal, daß ähnlich wie in den 60er und 70er Jahren überall auf der Welt neue Verfassungen entstehen und damit wiederum mehr Doktorarbeiten und Aufsatzthemen bereit liegen als vergeben werden können. Die Arbeitsmöglichkeiten sind dabei insgesamt besser als damals, das Internet eröffnet den direkten Zugriff auf die Verfassungsdiskussion in überseeischen Kontinenten, vor allem in Asien. Die wichtigsten englischsprachigen Zeitungen in Thailand z.B. sind on line verfügbar ${ }^{32}$ und berichten täglich ausführlich über den dort stattfindenden, mit breiter Öffentlichkeitsbeteiligung ausgetragenen Verfassungsgebungsprozeß, in sich selbst bereits ein erforschenswertes Phänomen.

Die naheliegende Frage ist natürlich, ob sich die Geschichte wiederholt, oder ob der demokratische Verfassungsstaat diesmal eine bessere Chance hat. Keines der grundlegenden Probleme, an der Demokratien in der Vergangenheit gescheitert sind, ist ja gelöst, der häufig unerfüllbare Imperativ wirtschaftlicher Leistung für Regierungen, die vor Wählern bestehen müssen, die Probleme majoritärer Demokratie in multi-ethnischen Staaten, extreme wirtschaftliche und soziale Ungleichheit, die zur Umsetzung wirtschaftlicher in politische Macht z.B. durch Korruption einlädt. Der Fundamentalismus ist als neuer Gegner hinzugekommen, bedroht den demokratischen Verfassungsstaat, wo er bereits gut integriert erschien, oder wo man optimistisch auf Demokratisierung hoffen konnte. Trotzdem sollte sich die Geschichte nicht einfach wiederholen. Ein wichtiger Unterschied ist, daß diktatorische Entwicklungswege gescheitert sind. In einem bemerkenswerten Perspektivenwechsel der Politikwissenschaft werden demokratischer Verfassungsstaat und Menschenrechte nicht mehr als Entwicklungshindernis oder als Ergebnis von Entwicklung, sondern als deren Bedingung diskutiert. ${ }^{33}$

\section{5. Überseeische Verfassungsvergleichung: Ende der Einbahnstraße}

Realistischerweise wird man aber davon auszugehen haben, daß die überseeische Verfassungsvergleichung nur dann aus ihrer marginalen Position in der deutschen Rechtswissenschaft herauskommen wird, wenn sich nachweisen läßt, daß das Studium überseeischer Verfassungsordnungen nicht nur Selbstzweck ist, sondern in ähnlicher Weise zur Ent- 
wicklung einer internationalen Verfassungsrechtswissenschaft beitragen kann, wie es der Verfassungsvergleich im europäischen-atlantischen Verfassungsraum längst tut. ${ }^{34}$

Zwei Aspekte der neuen Verfassungsgeneration könnten für Fortschritte in dieser Hinsicht sprechen: Die Bedeutung der Menschenrechte und der Verfassungsgerichtsbarkeit.

\section{a) Menschenrechte}

Die Bedeutung von Menschenrechten im Prozeß der Demokratisierungsbewegungen ist sicher ein ganz wesentliches Merkmal der neuen Entwicklung. Schon während der Herrschaft autoritärer Modelle entstanden überall in der Dritten Welt Menschenrechtsbewegungen, die unter Berufung auf die - von ihren Staaten jeweils gezeichneten, wenn auch nicht eingehaltenen - internationalen Mennschenrechtspakte für Veränderungen in ihren Heimatländern kämpften und die, gerade weil sie sich dabei auf internationale Dokumente beriefen, auch internationale Unterstützung mobilisieren konnten. ${ }^{35}$

Für die Verfassungsvergleichung sind zwei Aspekte bemerkenswert: Da bei der Formulierung von Grundrechtskatalogen im gegenwärtigen Demokratisierungsprozeß die internationalen Menschenrechtspakte eine wichtige Rolle spielen, ${ }^{36}$ entsteht eine weltweite Menschenrechtsdiskussion, die durch die internationalen Pakte bestimmt wird und auch weltweite Rezeptionsprozesse auslöst. Grundrechtsdogmatik wird in ungeahntem Umfang international, Auslegungsvorschläge und Fallrecht werden grenzüberschreitend diskutiert.

Das wirkt in beide Richtungen. Auch dort wo der Menschenrechtsdiskurs vor Ort schwach ist, kann mit Rückgriff auf internationale und ausländische Interpretationen argumentiert werden. Aber umgekehrt trägt die Tatsache, daß aus internationalen Pakten rezipierte Grundrechte nunmehr in vielen Ländern der Erde als nationales Recht bearbeitet und ausgelegt werden, auch zur Effektivität der völkerrechtlichen Pakte bei: Es entsteht auf diese Weise nämlich ein Fallrecht und eine Dogmatik, die die Berichtssysteme der UN-Pakte kaum zur Entstehung gebracht hätten, was für die Auslegung der internationalen Pakte

Für Europa: Bieber/Widmer (eds.), L'espace constitutionnel européen, 1995.

Dazu Ginther, Zivilgesellschaft und Entwicklung, VRÜ 1997, S. 133 ff.; (unvollständige) Übersicht über die Organisationsvielf alt: http://www.webcom.com/hrin/intlgrps.html

Kabudi, The Judiciary and Human Rights in Tanzania: Domestic Application of International Human Rights Norms, VRÜ 1991, S. 271 ff.; Human Rights Jurisprudence in East Africa: A Comparative Study of Fundamental Rights and Freedoms of the Individual in Tanzania, Kenya and Uganda, VRÜ-Beihefte 15, 1995; Scholler, Die neue äthiopische Verfassung und der Schutz der Menschenrechte, VRÜ 1997, S. 166 ff.; De Vos, The Role of International Human Rights Law in the Drafting of the Social and Economic Rights in South Africa's 1996 Constitution, Conference on Constitutional Transition, Hongkong 1997. 
nicht ohne Einfluß bleiben wird. Nicht nur für den Verfassungsvergleicher, auch für den Völkerrechtler werden überseeische Verfassungsordnungen damit unmittelbar relevant.

\section{b) Verfassungsgerichtsbarkeit}

Der zweite wichtige Aspekt der dritten Demokratisierungswelle ist die Bedeutung der Verfassungsgerichtsbarkeit. Ältere Diskussionen über die Vereinbarkeit von Demokratie und richterlichem Prüfungsrecht und Problematisierungen des gerichtlichen Schutzes wohlerworbener Rechte und entwicklungspolitischer Notwendigkeiten scheinen vergessen. Es war sicher auch der Erfolg der Verfassungsgerichtsbarkeit in der zweiten Demokratisierungswelle nach dem Krieg in Ländern wie Deutschland und Italien, die dazu führte, daß richterliche Kontrolle der Staatsgewalt in kaum einem Land der dritten Demokratisierungswelle fehlt. ${ }^{37}$ Man braucht die deutsche Neigung, Verfassungsrecht nur dann ernst zu nehmen, wenn es justitiabel ist, nicht zu teilen, um zu erkennen, daß die Justitiabilität dem Verfassungsrecht eine grundlegend andere Bedeutung gibt, und es nicht nur für den Wissenschaftler, sondern auch für den Praktiker interessanter macht.

Die Kombination weltweit sich durchsetzender vergleichbarer Grundrechtsstandards und deren Bearbeitung durch Verfassungsgerichte wird daher in Zukunft mehr als bisher eine Verfassungsrechtsentwicklung im Süden zur Folge haben, die nicht einfach nur als Exotik interessant ist, sondern die der Praktiker nicht ignorieren kann und die für den Wissenschaftler, der das eigene Fach nicht provinziell betreibt, ein Fülle von Anregungen für die eigene Arbeit enthält.

\section{c) Zwei Beispiele}

Dafür zum Abschluß zwei Beispiele:

Mein erstes Beispiel ist die Umweltrechtsprechung des indischen Supreme Court. Indien spielte während des ganzen Zeitraums, den wir betrachten, eine Sonderrolle. Es bewahrte, selbst während der emergency, seine demokratische Verfassungsstaatlichkeit und war immer ein Gegenbeweis gegen die These, daß Demokratie unter den Bedingungen eines Entwicklungslandes nicht funktionieren könnte. Bedenkt man, daß in der Maastricht-Dis- 
kussion die Möglichkeit europäischer Demokratie mit 13 Sprachen bezweifelt wird, ${ }^{38}$ sollte man einmal über die Leistung nachdenken, Demokratie über alle Krisen hinweg in einem Land mit über 200 Sprachen am Leben zu erhalten. Insofern ist es vielleicht ein wenig irreführend, dieses Beispiel im Zusammenhang mit neueren Entwicklungen zu bringen, aber für die Fruchtbarkeit überseeischer Verfassungsvergleichung und auch das Ignorieren wichtiger theoretischer und dogmatischer Lösungen, die dort gefunden worden sind, ist es doch besonders interessant.

Der indische Supreme Court und in seinem Gefolge andere Gerichte, haben sich in ungewöhnlichem Maße die Aufgabe gestellt, aktivistisch Defizite von Verwaltung und Politik auszugleichen und Verheißungen der indischen Verfassung, insbesondere den Grundrechtsteil, ernst zu nehmen. ${ }^{39}$ Vor allem seit den 80 er Jahren und nach der weltweit beachteten Umweltkatastrophe von Bophal ${ }^{40}$ hat der indische Gerichtshof der Verfassung immer schärfere Maßstäbe für die Umweltpolitik entnommen. Dabei konnte das Gericht an ein schon 1976 eingefügtes Staatsziel Umweltschutz anknüpfen. (Art. 48A: The State shall endeavour to protect and improve the environment and to safeguard the forest and wildlife of the country"). Gleichzeitig wurde eine Grundpflicht für den Bürger "to protect and improve the environment" (Art 51A) eingeführt. Vielleicht noch mehr Impetus erlangt diese Rechtsprechung aus der Mobilisierung des Grundrechts auf Leben und Gesundheit als Schutzpflichten ${ }^{41}$ und prozessual aus einer radikalen Öffnung der Grundrechtsklage und der Beschwerdebefugnis sowie der vom Gericht sich selbst zugebilligten Kompetenz, auch flexible Maßnahmen anzuordnen (in einem Urteil z.B. Umwelterziehung in den Schulen ${ }^{42}$ ). Umweltinitiativen, am erfolgreichsten der Umweltanwalt Mehta ${ }^{43}$, konnten daher mit Hilfe des Gerichts erhebliche Erfolge erzielen. Es wäre falsch zu behaupten, das sei international nicht beachtet worden - einige der genannten Urteile fanden ihren Weg bis in die Spalten deutscher Tageszeitungen -, aber eine Auseinandersetzung mit der Dogmatik verfassungsrechtlichen Umweltschutzes, die hier entwickelt wird und die angesichts deutscher Diskussionen über den juristischen Gehalt eines Staatsziels Umweltschutz durchaus auch in der Bundesrepublik von Interesse wäre, steht noch aus.

Grimm, Braucht Europa eine Verfassung?, JZ 1995, S. $581 \mathrm{ff}$.

Vandermay;, The Role of the Judiciary in India's Constitutional Democracy, Hastings Int'l \& Comp.L.Rev 20 (1996), S. $103 \mathrm{ff}$.

Abraham/Abraham, The Bophal Case and the Development of Environmental Case Law in India, Int.Comp.Law Q 40 (1991), S. 335 ff.

Abraham/Abraham, (Fn. 40), S. 360.

MC Mehta v. Union of India and Others, Supreme Court Reports [1988] 2 S.C.R v. 12.1.1988 (http://www.igc.apc.org/elaw/asia/india/mehta-v-union-jan-88.html.).

Zu ihm vgl. http://www.igc.apc.org/elaw/asia/india/icela.html. 
Das zweite Beispiel ist die Rolle des südafrikanischen Constitutional Court im Prozeß des Systemwechsels vom Apartheidsystem zur Demokratie. Dieses Beispiel ist doppelt aufschlußreich für die Fruchtbarkeit "überseeischer" Verfassungsvergleichung. Zum einen belegt es nochmals dramatisch den Perspektivenwechsel im Süden, die nunmehr fast schon zu unkritische Übernahme verfassungsstaatlicher Modelle, die von den handelnden Akteuren noch vor wenigen Jahren als "bürgerlich" abgelehnt worden wären, zum anderen kann aber auch der südafrikanische Gerichtshof, wie der indische, Beachtung weit über Südafrika hinaus für jede nicht eurozentrische Verfassungsvergleichung beanspruchen. Die südafrikanische Verfassungsentwicklung hat ihm nämlich eine völlig neuartige Rolle im Prozeß der Verfassungsgebung zugewiesen:

Die Aufgabe war, den zwischen altem Regime und ANC friedlich ausgehandelten Systemwechsel in eine neue Verfassungsordnung zu überführen, die gleichzeitig die vereinbarten Verfassungsprinzipien wahrte und dem Prinzip der verfassunggebenden Gewalt des Volkes gerecht wurde $^{44}$. Der Gerichtshof beschreibt dieses Problem in seinem First Certification judgment $^{45}$ (das es verdient hat, ein Klassiker der Verfassungsvergleichung zu werden). Die folgenden Ausführungen können wörtlich zitiert werden.:

[13] The impasse was resolved by a compromise which enabled both sides to attain their basic goals without sacrificing principle. ... Instead of an outright transmission of power from the old order to the new, there would be a programmed two-stage transition. An interim government ... would govern the country on a coalition basis while a final constitution was being drafted. A national legislature, elected (directly and indirectly) by universal adult suffrage, would double as the constitution-making body and would draft the new constitution within a given time. But - and herein lies the key to the resolution of the deadlock - that text would have to comply with certain guidelines agreed upon in advance by the negotiating parties. What is more, an independent arbiter would have to ascertain and declare whether the new constitution indeed complied with the guidelines before it could come into force.

Dieser Schiedsrichter sollte der Constitutional Court sein.

$\mathrm{Daß}$ ein Gericht für diese Aufgabe ausgewählt wurde, ist nicht selbstverständlich. Schließlich waren auch die Gerichte Teil des Apartheid-Systems gewesen. Aber während dieses System für die schwarze Mehrheit als Gewaltherrschaft funktionierte, konnten im weißen politischen und Rechtssystem Spuren rechtsstaatlicher Tradition überleben. Abel hat darge-

Klug, South Africa's New Constitution: the Challenge of Diversity and Identity, VRÜ 1995, S. $417 \mathrm{ff}$.

45

Constitutional Court CCT 23/1996, para 12 ff. (http://www.law.wits.ac.za/judments/certsum.html) 
stellt, wie dieses System gerade noch genug rechtsstaatliche Elemente enthielt, daß es sich von der Opposition als "politics by other means" ${ }^{46}$ einsetzen ließ, und zwar um so mehr, je näher das Ende des Apartheid-Regimes heranrückte. Außerdem wurde die Schiedsrichteraufgabe ja nicht einfach der Justiz (das wäre wohl politisch unmöglich gewesen), sondern einem Verfassungsgericht übertragen, das sorgfältig und ausgewogen zusammengesetzt werden konnte.

Die beschriebene Aufgabe und die vom Gerichtshof angewandte Methode machen das Urteil nun heute schon zu einem klassischen Text für den Verfassungsvergleicher: Das Gericht muß ja die neue Verfassung an Verfassungsprinzipien überprüfen, eine Aufgabe, die sich wohl seriös nur so bewältigen läßt, wie das Gericht es tut, nämlich, indem es verfassungsvergleichend nach der Bandbreite fragt, in der die entsprechenden Prinzipien weltweit in demokratischen Verfassungsstaaten erfüllt sind, und dann prüft, ob die südafrikanische Lösung sich in diesen Rahmen einpaßt oder ihn verläßt. Insofern stellt dieses Urteil eine Fundgrube dar, da wir für eine Fülle von Grundrechten und Verfassungsprinzipien eine Übersicht über ihre weltweite Verwirklichung finden.

Insgesamt kommt der Gerichtshof mit gesundem judicial restraint zur Antwort, daß die neue Verfassung sich im Rahmen der Prinzipien hält, allerdings wird die Certifizierung wegen einiger Mängel verneint, was auch verfassungspolitisch sinnvoll ist, da eine uneingeschränkte Bestätigung bereits in der ersten Runde allzu leicht den Eindruck erweckt hätte, daß das Gericht sich vor den politischen Instanzen duckt. Nach einigen Nachbesserungen hat das Gericht die neue Verfassung dann bestätigt.

Ich komme zum Schluß.

Der Zeitschrift Verfassung und Recht in Übersee ist offensichtlich 30 Jahre nach ihrer Gründung das Thema nicht verloren gegangen. Ganz im Gegenteil drängen sich überall im Beobachtungsraum neue verfassungsrechtliche Entwicklungen auf. Herbert Krüger beendete seinen eingangs gewürdigten Einleitungsaufsatz mit einem "Aufruf zur Mitarbeit". Diesen gilt es heute zu erneuern. 


\section{ABSTRACTS}

\section{Three Decades of Comparative Constitutional Law and Development}

By Brun-Otto Bryde

"Verfassung und Recht in Übersee" was founded at a time when development through modern law and Western constitutionalism seemed to be a realistic aim for the immediate future. When the prospects of constitutionalism in the Third World turned bleak, (constitutional) law and development studies were in danger of loosing their subject. The third wave of democratisation gives hope for a fresh start.

Though the major problems for viable democratic government in developing countries are not overcome, the bankruptcy of authoritarian models of development might give democracy and rule of law a better chance this time.

Of special importance is the role of human rights and the increasing popularity of constitutional courts. Both developments should lead to an internationalisation of constitutional law discourse in which the North should be ready to appreciate better the contribution of the South to this discourse. The jurisprudence of the Indian Supreme Court in environmental law and the role of South African Constitutional Court in constitutional transition are examples.

\section{Public International Law and Overseas Nations}

\section{By Philip Kunig}

The initials VRÜ have always given room for the interpretation that the Journal Verfassung und Recht in Übersee deals with public international law in overseas nations ("Völkerrecht in Übersee"). And, indeed, from the very beginning, the Journal not only published articles on comparative constitutional law but laid a strong emphasis on public international law. The article first recalls some of the highlights of international law "before" VRÜ, then goes on to describe the situation in international law at the time of the first issue of this Journal and, thirdly, focuses on aspects of "international law and overseas" in present and future times. The Journal has always taken time to look back to the times which were characterized by the dichotomy of "civilized" and "uncivilized" nations. However, as the Journal came into existence after the period of decolonization was - almost totally - over, special attention has been given to the situation of the newly independent states within the inter- 